\title{
Synthesis, Biological Activity of Salidroside and Its Analogues
}

\author{
Yibing Guo, ${ }^{a, c, \#}$ Yahong ZhaO, ${ }^{a, \#}$ Cheng Zheng, ${ }^{b}$ Ying Meng, ${ }^{a}$ and Yumin YAnG ${ }^{*, a}$ \\ ${ }^{a}$ Jiangsu Key Laboratory of Neuroregeneration, Nantong University; ${ }^{b}$ Institue of Nautical Medicine, Nantong University; \\ and ${ }^{c}$ Medical School, Nantong University; No. 19, Qixiu Road, Nantong, Jiangsu 226001, P. R. China. \\ Received April 2, 2010; accepted September 15, 2010; published online September 30, 2010
}

Salidroside is a phenylpropanoid glycoside isolated from Rhodiola rosea $\mathrm{L}$., a traditional Chinese medicinal plant, and has displayed a broad spectrum of pharmacological properties. In this paper, about 18 novel salidroside analogues were prepared through Koenigs-Knorr method, the effects of these compounds over PC12 was assessed with the 3-(4,5-dimethylthiazol-2-yl)-2,5-diphenyltetrazolium bromide (MTT) method. The novel compounds differ in the substituents attached to the benzene ring or in the glycosyl donor. According to the data, compounds (3,5-dimethoxyphenyl)methyl $\beta$-D-glucopyranoside and (3,5-dimethoxyphenyl)methyl $\beta$-D-galactopyranoside with methoxy group at 3 and 5-positions of the benzene ring were the most viability at concentration of $300 \mu \mathrm{mol} / \mathrm{l}$ and $60 \mu \mathrm{mol} / \mathrm{l}$, respectively.

Key words salidroside; analogue; 3-(4,5-dimethylthiazol-2-yl)-2,5-diphenyltetrazolium bromide method; hypoglycemia and serum limitation; PC12 cell

Salidroside, a traditional Chinese medicine, was known as the main bioactive component in the root of Rhodiola sachalinensis A. BoR., and was reported to have many pharmacological properties, such as resisting anoxia, ${ }^{1)}$ anti-radiation and antifatigue, ${ }^{2,3}$ improving oxygen lack and postponing ageing. ${ }^{4)}$ In recent years, salidroside has been used in such special posts as diver, astronaut, pilot and mountaineer to enhance the ability for survival in adverse environment. ${ }^{5)}$ Furthermore, many researches revealed that it has the activities of preventing cardiovascular disease ${ }^{6)}$ and anti-tumor. ${ }^{7)}$ However, the resource of wild $R$. sachalinensis A. BoR. was on the edge of exhaustion.

Salidroside is a 2-(4-hydroxyphenyl)ethyl $\beta$-D-glucopyranoside (Fig. 1, 5e) which may be synthesized by glucosylation of tyrosol. ${ }^{8,9)}$ Considerable effort has been devoted to the isolation and assess of pharmacological properties, synthesis and structure modification of salidroside is very few. In order

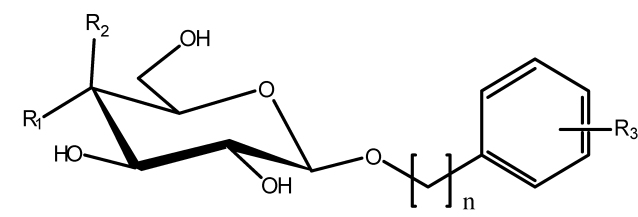

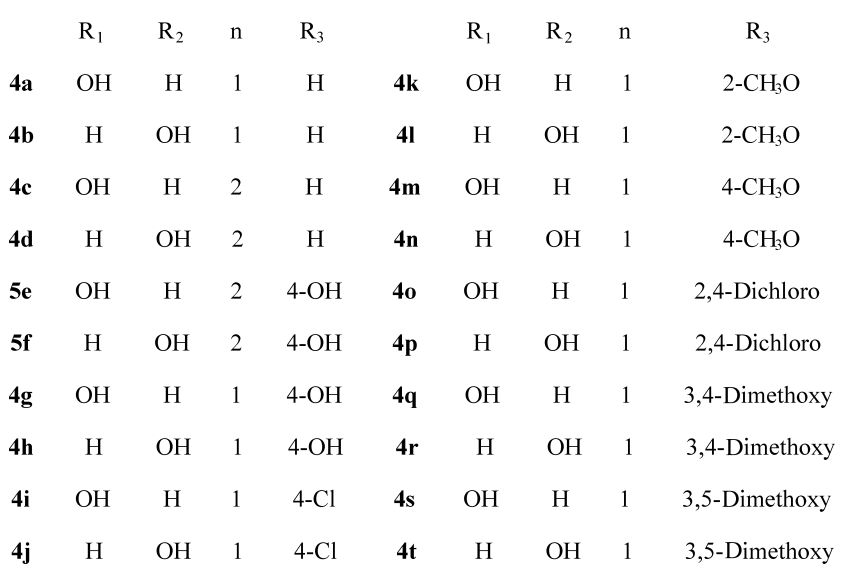

Fig. 1. Structure of Salidroside and Its Analogues to search for more activity compounds, 20 compounds (Fig. $1, \mathbf{4 a}-\mathbf{d}, \mathbf{5} \mathbf{e}-\mathbf{f}, \mathbf{4 g}-\mathbf{t})$ were successfully produced by Koenigs-Knorr method.

The synthesis of the compounds $\mathbf{3 a}-\mathbf{t}$ was completed, which starting from the corresponding acetobromo-sugars (D-glucose, D-galactose) with the suitable substituted aromatic alcohol. The reaction was conducted in dry ethyl ether and dichloromethane for $10 \mathrm{~h}$ under dark with the presence of $\mathrm{Ag}_{2} \mathrm{CO}_{3}$. The process was optimized in the presence of $4 \AA$ MS. Powdered MS $4 \AA$ was dried at $140{ }^{\circ} \mathrm{C}$ under reduced pressure for $1 \mathrm{~d}$ before use. The designed target compounds $\mathbf{4 a}-\mathbf{4 t}$ were obtained from $3 \mathbf{a}-\mathbf{4 t}$ by direct deacetylation with $\mathrm{CH}_{3} \mathrm{ONa} / \mathrm{CH}_{3} \mathrm{OH}$ in good yields (Chart 1, over $90 \%$ to almost in quantitative). The catalytic hydrogenation of $4 \mathbf{e}$ and $\mathbf{f}$ to remove the protection of benzyl in the phenol hydroxy group is operated by refluxed with $5 \% \mathrm{Pd} / \mathrm{C}$ and $\mathrm{HCOONH}_{4}$ in anhydrous methanol (Chart 1).

According to 3-(4,5-dimethylthiazol-2-yl)-2,5-diphenyltetrazolium bromide (MTT) assay, after the PC12 cells were exposed to hypoglycemia and serum limitation for $24 \mathrm{~h}$, it was found that hypoglycemia and serum limitation induced significant decreases in cell viability of cultured PC12 cells, as compared to that of control group. Exposure to hypoglycemia and serum limitation for $24 \mathrm{~h}$ was used to induce PC12 cell injury in all experiments unless otherwise stated. The cell viability of PC12 for each compound provide a quantitative comparison of salidroside and its analogues. MTT assay confirmed that the exposure to hypoglycemia and serum limitation caused the cell viability loss in cultured PC12 cells, and further showed that pretreatment with salidroside and its analogues at different concentrations (60, $300,1000 \mu \mathrm{mol} / 1)$ significantly attenuated the cell viability loss evoked by hypoglycemia and serum limitation except $\mathbf{4 i}$ and $\mathbf{4 j}$, with $60 \mu \mathrm{mol} / 1 \mathbf{4 t}$ and $300 \mu \mathrm{mol} / 1 \mathrm{4s}$ yielding an attenuating effect close to that of control group. Overall, the data indicated that $\mathbf{4 s}$ and $\mathbf{4 t}$ are more active in the assay, which provided some insights into the design of new salidroside analogue.

\section{Experimental}

General Commercial reagents were used without further purification unless otherwise stated. All melting points are uncorrected. ${ }^{1} \mathrm{H}-\mathrm{NMR}$ spectra 


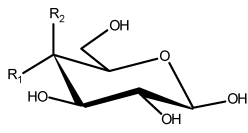

1a $\mathrm{R}_{1}=\mathrm{OH}, \mathrm{R}_{2}=\mathrm{H}$ 1b $\mathrm{R}_{1}=\mathrm{H}, \mathrm{R}_{2}=\mathrm{OH}$

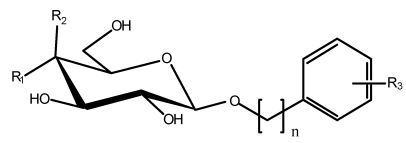

4a-4t $\mathrm{R}_{1}=\mathrm{OH}, \mathrm{R}_{2}=\mathrm{H}$ or $\mathrm{R}_{1}=\mathrm{H}, \mathrm{R}_{2}=\mathrm{OH}$ $n=1$ or $2 R_{3}=$ substituted group

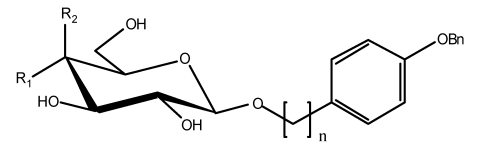

$4 e-4 f$

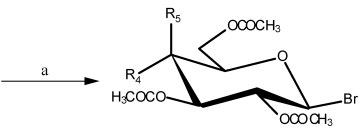

2a $\mathrm{R}_{4}=\mathrm{OCOCH}_{3}, \mathrm{R}_{5}=\mathrm{H}$ 2b R $4=\mathrm{H}, \mathrm{R}_{5}=\mathrm{OCOCH}_{3}$

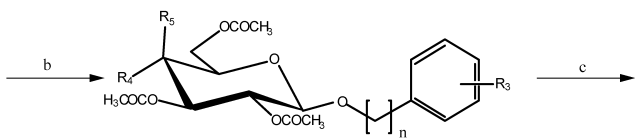

3a-3t $\mathrm{R}_{4}=\mathrm{OCOCH}_{3}, \mathrm{R}_{5}=\mathrm{H}$ or $\mathrm{R}_{4}=\mathrm{H}, \mathrm{R}_{5}=\mathrm{OCOCH}_{3}$, $\mathrm{n}=1$ or $2 \mathrm{R}_{3}=$ substituted group

Reagents: a) $\mathrm{P}, \mathrm{Br}_{2}, \mathrm{Ac}_{2} \mathrm{O}$; b) $\mathrm{Ag}_{2} \mathrm{CO}_{3}, \mathrm{CH}_{2} \mathrm{Cl}_{2}, \mathrm{Et} 2 \mathrm{O}$, rt, 10 h; c) NaOMe, $\mathrm{MeOH}, \mathrm{rt}, 3$ h; d) $\mathrm{HCOONH}_{4}, 5 \%$ Pd/C, $\mathrm{CH}_{3} \mathrm{OH}, \mathrm{reflux}, 6$ h.

Chart 1. Synthetic Route for Salidroside and Its Analogues

Table 1. Experiment Data of Compounds

\begin{tabular}{|c|c|c|c|c|c|c|c|c|c|c|c|}
\hline $\begin{array}{c}\text { Compound } \\
\text { No. }\end{array}$ & $\begin{array}{c}\text { Yield } \\
(\%)\end{array}$ & $\begin{array}{c}\text { Compound } \\
\text { No. }\end{array}$ & $\begin{array}{l}\text { Yield } \\
(\%)\end{array}$ & $\begin{array}{l}\mathrm{mp} \\
\left({ }^{\circ} \mathrm{C}\right)\end{array}$ & $\begin{array}{c}\delta \\
(\mathrm{C} 1-\mathrm{H})\end{array}$ & $\begin{array}{c}\text { Compound } \\
\text { No. }\end{array}$ & $\begin{array}{l}\text { Yield } \\
(\%)\end{array}$ & $\begin{array}{c}\text { Compound } \\
\text { No. }\end{array}$ & $\begin{array}{l}\text { Yield } \\
(\%)\end{array}$ & $\begin{array}{l}\mathrm{mp} \\
\left({ }^{\circ} \mathrm{C}\right)\end{array}$ & $\begin{array}{c}\delta \\
(\mathrm{C} 1-\mathrm{H})\end{array}$ \\
\hline $3 \mathbf{a}$ & 52 & $4 a$ & 100 & $120-121$ & $4.18(J=7.8 \mathrm{~Hz})$ & $3 \mathbf{k}$ & 70 & $4 k$ & 96 & $121-122$ & $4.5(J=7.85 \mathrm{~Hz})$ \\
\hline $\mathbf{3 b}$ & 62 & $4 b$ & 96 & $80-82$ & $4.18(J=7.7 \mathrm{~Hz})$ & 31 & 72 & 41 & 91 & $142-143$ & $4.46(J=7.9 \mathrm{~Hz})$ \\
\hline $3 c$ & 66 & $4 c$ & 92 & $96-97$ & $4.17(J=7.8 \mathrm{~Hz})$ & $3 \mathrm{~m}$ & 68 & $4 m$ & 92 & $149-150$ & $4.5(J=8.05 \mathrm{~Hz})$ \\
\hline 3d & 61 & $4 d$ & 94 & $97-98$ & $4.13(J=7.2 \mathrm{~Hz})$ & $3 n$ & 58 & $4 n$ & 95 & $130-131$ & $4.45(J=7.85 \mathrm{~Hz})$ \\
\hline $3 e$ & 58 & $5 e$ & 91 & $159-160$ & $4.15(J=7.8 \mathrm{~Hz})$ & 30 & 55 & 40 & 90 & $160-161$ & $4.52(J=7.89 \mathrm{~Hz})$ \\
\hline $3 f$ & 60 & $5 f$ & 100 & $55-57$ & $4.10(J=7.25 \mathrm{~Hz})$ & $3 p$ & 62 & $4 p$ & 96 & - & $4.46(J=7.47 \mathrm{~Hz})$ \\
\hline $3 g$ & 61 & $4 \mathrm{~g}$ & 100 & $164-165$ & $4.38(J=7.32 \mathrm{~Hz})$ & $3 q$ & 35 & $4 q$ & 94 & $117-118$ & $4.51(J=8.0 \mathrm{~Hz})$ \\
\hline $3 \mathbf{h}$ & 64 & $4 h$ & 100 & $137-139$ & $4.43(J=7.56 \mathrm{~Hz})$ & $3 \mathbf{r}$ & 42 & $4 r$ & 93 & $146-148$ & $4.45(J=8.0 \mathrm{~Hz})$ \\
\hline $3 \mathbf{i}$ & 55 & $4 i$ & 96 & $136-138$ & $4.53(J=7.2 \mathrm{~Hz})$ & $3 s$ & 63 & $4 s$ & 95 & $146-148$ & $4.51(J=8.0 \mathrm{~Hz})$ \\
\hline $3 \mathbf{j}$ & 52 & $4 j$ & 92 & $138-140$ & $4.45(J=6.85 \mathrm{~Hz})$ & $3 t$ & 65 & $4 t$ & 92 & $150-152$ & $4.45(J=8.0 \mathrm{~Hz})$ \\
\hline
\end{tabular}

were recorded on a Bruker AC 400 instrument at $500 \mathrm{MHz}$, and the following abbreviations are used for the description of the patterns: $s=$ singlet, $\mathrm{d}=$ doublet, $\mathrm{t}=$ triplet, $\mathrm{m}=$ multiplet. The ${ }^{1} \mathrm{H}-\mathrm{NMR}$ spectra were recorded with tetramethylsilane (TMS, $\delta 0.00$ ) as the internal standard and were run in dimethyl sulfoxide (DMSO) or $\mathrm{D}_{2} \mathrm{O}, J$ values are expressed in $\mathrm{Hz}$. Flash chromatography was performed on silica gel (200 - 300 mesh) (Hai Yang Chemical Factory, Qingdao, Shangdong, P. R. China). Compounds 2a and b were prepared through literature. ${ }^{10)}$

General Procedure for Compounds $4 \mathbf{a}-\mathbf{t}$ A mixture of $\mathbf{2 a}$ or $\mathbf{2 b}$ $(2.05 \mathrm{~g}, 5 \mathrm{mmol})$ and alcohol $(4 \mathrm{mmol})$ was dissolved in dry dichloromethane-ether $(2: 1,15 \mathrm{ml})$ and $2 \mathrm{~g} 4 \AA \mathrm{MS}$ was added, then stirred at room temperature under $\mathrm{N}_{2}$ atmosphere for $20 \mathrm{~min}$. After that $\mathrm{Ag}_{2} \mathrm{CO}_{3}(1.4 \mathrm{~g}$, $5 \mathrm{mmol}$ ) was added rapidly to the mixture and stirring continued for $10 \mathrm{~h}$ avoid light. The reaction mixture was filtered and evaporated under reduced pressure. The crude product was purified by column chromatography using $1: 3$ or $1: 2$ EtOAc-petroleum ether to afford a colourless gummy mass 3a-t (yield shown in Table 1). To a solution of compounds $\mathbf{3 a}-\mathbf{t}(2.5$ $\mathrm{mmol})$ in $\mathrm{MeOH}(10 \mathrm{ml})$ was added $\mathrm{NaOMe}(10 \mathrm{mg})$, and the reaction mixture was stirred at room temperature for $2 \mathrm{~h}$. After that diluted with $\mathrm{MeOH}$ and acidified with Amberlite IRA-120 $(\mathrm{H}+)$ resin to $\mathrm{pH}=7$. The reaction mixture was filtered and evaporated to dryness under reduced pressure afford $\mathbf{4 a -} \mathbf{t}$ as white solid ${ }^{11)}$ (Table 1 ).

Procedure for Compounds 5e and f 2-(4-Hydroxyphenyl)ethyl $\beta$-DGlucopyranoside (5e): A mixture of $4 \mathrm{e}(180 \mathrm{mg}, 460 \mu \mathrm{mol}), 5 \% \mathrm{Pd} / \mathrm{C}$ $(208 \mathrm{mg})$ and ammonium formate $(0.15 \mathrm{~g}, 2.3 \mathrm{mmol})$ in $\mathrm{MeOH}(6 \mathrm{ml})$ was stirred at refluxing for $6 \mathrm{~h}$. Then filtered, concentrated and purified by column chromatography using $8: 1 \mathrm{CHCl}_{3}-\mathrm{MeOH}$ to give $125 \mathrm{mg}(91 \%)$ of $\mathbf{5 e}$. The physical constants were in agreement with the literature. ${ }^{13)}$

2-(4-Hydroxyphenyl)ethyl $\beta$-D-Galactopyranoside (5f): 5 f was prepared from $\mathbf{4 f}$ in the same manner just as described above, white solid: ${ }^{1} \mathrm{H}-\mathrm{NMR}$
(DMSO) $\delta: 9.12(1 \mathrm{H}, \mathrm{s}), 7.02(2 \mathrm{H}, \mathrm{d}, J=8.35 \mathrm{~Hz}), 6.66(2 \mathrm{H}, \mathrm{d}, J=6.55 \mathrm{~Hz})$, $4.75(1 \mathrm{H}, \mathrm{s}), 4.62(1 \mathrm{H}, \mathrm{s}), 4.51(1 \mathrm{H}, \mathrm{s}), 4.28(1 \mathrm{H}, \mathrm{s}), 4.10(1 \mathrm{H}, \mathrm{d}$, $J=7.25 \mathrm{~Hz}), 3.82-3.87(1 \mathrm{H}, \mathrm{m}), 3.62(1 \mathrm{H}, \mathrm{s}), 3.45-3.58(3 \mathrm{H}, \mathrm{m}), 3.27-$ $3.32(3 \mathrm{H}, \mathrm{m}), 2.70-2.74(2 \mathrm{H}, \mathrm{m})$. IR (KBr) cm $\mathrm{cm}^{-1}: 3268,1630,1614,1246$, 1079, 1020. Anal. Calcd for $\mathrm{C}_{14} \mathrm{H}_{20} \mathrm{O}_{7}:$ C, 55.99; H, 6.71. Found: C, 56.08; H, 6.77.

Cell Culture Rat PC-12 cells, obtained from the American Type Culture Collection (Manassas, VA, U.S.A.), were plated and maintained in highglucose Dulbecco's modified Eagle's medium (DMEM) supplemented with $10 \%$ horse serum, $5 \%$ fetal bovine serum (FBS), $100 \mathrm{U} / \mathrm{ml}$ penicillin, and $100 \mathrm{U} / \mathrm{ml}$ streptomycin in $5 \% \mathrm{CO}_{2} /$ air at $37^{\circ} \mathrm{C}$. After being pretreated with 60,300 , or $1000 \mu \mathrm{mol} / 1$ compounds synthesized above for $24 \mathrm{~h}$, the cells were subjected to hypoglycemia and serum limitation by replacing the culture medium with the glucose-free DMEM supplement with $1 \%$ horse serum and $1 \% \mathrm{FBS}, 100 \mathrm{U} / \mathrm{ml}$ penicillin, and $100 \mathrm{U} / \mathrm{ml}$ streptomycin, and in the presence of compounds at original concentrations for another $24 \mathrm{~h}$ incubation. ${ }^{14)}$ At the end of incubation, the MTT solution was added and further incubated at $37^{\circ} \mathrm{C}$ for $4 \mathrm{~h}$ followed by the addition of DMSO to dissolve the resulting formazan. The absorbance (optical density (OD)) values were measured by spectrophotometry at $570 \mathrm{~nm}$ with an EIX-800 Microelisa reader (Bio-Tek Inc., U.S.A.).

Acknowledgments The financial supports of Hi-Tech Research and Development Program of China (863 Program, Grant no.2006AA02A128), Nature Science Foundation of China (Grant no. 30970713), Basic Research Program of Jiangsu Province (Grant no. BK2009518) and Program for New Century Excellent Talents in University are gratefully acknowledged.

\section{References and Notes}

1) Chen Y. M., Chen J., Mao G. M., Fu J. Y., Zhejiang Prev. Med., 19, 
92-93 (2007).

2) Tong Q. H., Tan Z. S., Yang Q., J. Health Toxicol., 3, 183-184 (2004).

3) Luo C. H., Shu R., Gao Y., Modern Appl. Med., 13, 5-7 (1996).

4) Saratikov A. S., Die Pharmazie, 69, 394-398 (1968).

5) Ming H. Q., Xia G. C., Zhang R. D., Chin. Traditional Herb. Drugs, 19, 229-234 (1988)

6) Bonanome A., Pagnan A., Caruso D., Nutr. Metab. Cardiovasc. Dis., 10, 111-120 (2000)

7) Udintsev S. N., Schakhov V. P., Eur. J. Cancer, 27, 1182 (1991).

8) Troshchenko J., Khim. Prir. Soedin, 5, 256-260 (1969).

9) Ming H. Q., Chin. J. Pharm. Bull. 21, 373-375 (1986).

10) Barczai Martos M., Korosy F., Nature (London), 165, 369 (1950).

11) Benzyl $\beta$-D-galactopyranoside (4b): ${ }^{1} \mathrm{H}-\mathrm{NMR}$ (DMSO) $\delta$ : 7.38-7.40 $(2 \mathrm{H}, \mathrm{d}, J=6.95 \mathrm{~Hz}), 7.30-7.35(2 \mathrm{H}, \mathrm{m}), 7.25-7.28(1 \mathrm{H}, \mathrm{m}), 4.89(1 \mathrm{H}$ d, $J=4.85 \mathrm{~Hz}), 4.79(1 \mathrm{H}, \mathrm{d}, J=12.30 \mathrm{~Hz}), 4.65(1 \mathrm{H}, \mathrm{d}, J=5.45 \mathrm{~Hz})$, $4.53-4.58(2 \mathrm{H}, \mathrm{m}), 4.33(1 \mathrm{H}, \mathrm{d}, J=4.70 \mathrm{~Hz}), 4.18(1 \mathrm{H}, \mathrm{d}, J=7.70 \mathrm{~Hz})$ $3.64(1 \mathrm{H}, \mathrm{t}, J=3.65 \mathrm{~Hz}), 3.49-3.57(2 \mathrm{H}, \mathrm{m}), 3.25-3.27(1 \mathrm{H}, \mathrm{m})$. IR $(\mathrm{KBr}) \mathrm{cm}^{-1}: 3412,2943,1650,1454,1418,1211,1141,1077,1040$ Anal. Calcd for $\mathrm{C}_{13} \mathrm{H}_{18} \mathrm{O}_{6}$ : C, 57.77; $\mathrm{H}, 6.71$. Found: $\mathrm{C}, 57.68 ; \mathrm{H}, 6.80$. Phenethyl $\beta$-D-glucopyranoside (4c): ${ }^{1} \mathrm{H}$-NMR (DMSO) $\delta: 7.25-7.29$ $(4 \mathrm{H}, \mathrm{m}), 7.17-7.20(1 \mathrm{H}, \mathrm{m}), 3.91-3.96(1 \mathrm{H}, \mathrm{m}), 3.62-3.68(2 \mathrm{H}$, $\mathrm{m}), 3.40-3.45(1 \mathrm{H}, \mathrm{m}), 4.91(1 \mathrm{H}, \mathrm{d}, J=4.95 \mathrm{~Hz}), 4.86(1 \mathrm{H}, \mathrm{d}$ $J=4.86 \mathrm{~Hz}), 4.83(1 \mathrm{H}, \mathrm{d}, J=5.10 \mathrm{~Hz}), 4.43(1 \mathrm{H}, \mathrm{t}, J=6.0 \mathrm{~Hz}), 4.17$ $(1 \mathrm{H}, \mathrm{d}, J=7.80 \mathrm{~Hz}), 3.11-3.15(1 \mathrm{H}, \mathrm{m}), 3.07-3.10(1 \mathrm{H}, \mathrm{m}), 3.01-$ $3.05(1 \mathrm{H}, \mathrm{m}), 2.93-2.98(1 \mathrm{H}, \mathrm{m}), 2.83-2.87(2 \mathrm{H}, \mathrm{m})$. IR $(\mathrm{KBr})$ $\mathrm{cm}^{-1}: 3362,1940,1610,1515,1210,1072,1030$. Anal. Calcd for $\mathrm{C}_{14} \mathrm{H}_{20} \mathrm{O}_{6}:$ C, 59.14; H, 7.09. Found: C, 58.98; H, 7.12. 2-(4-Benzyloxyphenyl)ethyl $\beta$-D-glucopyranoside (4e): mp 96-98 ${ }^{\circ} \mathrm{C} .{ }^{1} \mathrm{H}-\mathrm{NMR}$ datum was in agreement with the literature. ${ }^{12)} \mathrm{IR}(\mathrm{KBr}) \mathrm{cm}^{-1}: 3419$, 2913, 2865, 1513, 1453, 1251, 1160, 1076, 1027. Anal. Calcd for $\mathrm{C}_{21} \mathrm{H}_{26} \mathrm{O}_{7}: \mathrm{C}, 64.6 ; \mathrm{H}, 6.71$. Found: C, 64.72; H, 6.58. 2-(4-Benzyloxyphenyl)ethyl $\beta$-D-galactopyranoside (4f): $\mathrm{mp} 127-128^{\circ} \mathrm{C} .{ }^{1} \mathrm{H}-$ NMR (DMSO) $\delta: 7.43(2 \mathrm{H}, \mathrm{d}, J=6.8 \mathrm{~Hz}), 7.36-7.39(2 \mathrm{H}, \mathrm{m}), 7.30$ $7.33(1 \mathrm{H}, \mathrm{m}), 7.17(2 \mathrm{H}, \mathrm{d}, J=8.7 \mathrm{~Hz}), 6.91(2 \mathrm{H}, \mathrm{d}, J=8.7 \mathrm{~Hz}), 5.06$
$(2 \mathrm{H}, \mathrm{s}), 4.75(1 \mathrm{H}, \mathrm{d}, J=4.50 \mathrm{~Hz}), 4.61(1 \mathrm{H}, \mathrm{d}, J=5.10 \mathrm{~Hz}), 4.50(1 \mathrm{H}, \mathrm{t}$, $J=5.30 \mathrm{~Hz}), 4.27(1 \mathrm{H}, \mathrm{d}, J=4.65 \mathrm{~Hz}), 4.11(1 \mathrm{H}, \mathrm{d}, J=7.25 \mathrm{~Hz}), 3.84$ $3.89(1 \mathrm{H}, \mathrm{m}), 3.56-3.62(2 \mathrm{H}, \mathrm{m}), 3.46-3.53(2 \mathrm{H}, \mathrm{m}), 3.30-3.33$ $(1 \mathrm{H}, \mathrm{m}), 3.25-3.28(2 \mathrm{H}, \mathrm{m}), 2.77-2.79(2 \mathrm{H}, \mathrm{m}) . \mathrm{IR}(\mathrm{KBr}) \mathrm{cm}^{-1}$. $3429,2934,1614,1514,1453,1252,1145,1058$. Anal. Calcd for $\mathrm{C}_{21} \mathrm{H}_{26} \mathrm{O}_{7}: \mathrm{C}, 64.6$; H, 6.71. Found: C, 64.52; H, 6.80. 2-(4-Hydroxyphenyl)methyl $\beta$-D-galactopyranoside $(\mathbf{4 h})$ : ${ }^{1} \mathrm{H}-\mathrm{NMR}\left(\mathrm{D}_{2} \mathrm{O}\right) \delta: 7.35$ $(2 \mathrm{H}, \quad$ d,$J=8.37 \mathrm{~Hz}), 6.91(2 \mathrm{H}, \quad \mathrm{d}, J=8.37 \mathrm{~Hz}), 4.85(1 \mathrm{H}, \mathrm{d}$, $J=11.28 \mathrm{~Hz}), 4.65(1 \mathrm{H}, \mathrm{d}, J=11.28 \mathrm{~Hz}), 4.43(1 \mathrm{H}, \mathrm{d}, J=7.56 \mathrm{~Hz}), 3.91$ $(1 \mathrm{H}, \mathrm{d}, J=3.03 \mathrm{~Hz}), 3.73-3.85(2 \mathrm{H}, \mathrm{m}), 3.59-3.69(2 \mathrm{H}, \mathrm{m}), 3.49-$ $3.55(1 \mathrm{H}, \mathrm{m})$. IR $(\mathrm{KBr}) \mathrm{cm}^{-1}: 3438,1250,1162,1040$. Anal. Calcd for $\mathrm{C}_{13} \mathrm{H}_{18} \mathrm{O}_{7}$ : C, 54.54; H, 6.34. Found: C, 54.62; H, 6.48. (4Chlorophenyl)methyl $\beta$-D-glucopyranoside (4i): ${ }^{1} \mathrm{H}-\mathrm{NMR}\left(\mathrm{D}_{2} \mathrm{O}\right) \delta$ : $7.47(4 \mathrm{H}, \mathrm{s}), 4.93(1 \mathrm{H}, \mathrm{d}, J=11.55 \mathrm{~Hz}), 4.53(1 \mathrm{H}, \mathrm{d}, J=7.20 \mathrm{~Hz}), 3.93$ $(1 \mathrm{H}, \mathrm{d}, J=11.55 \mathrm{~Hz}), 3.76(2 \mathrm{H}, \mathrm{s}), 3.41-3.46(4 \mathrm{H}, \mathrm{m}) . \mathrm{IR}(\mathrm{KBr})$ $\mathrm{cm}^{-1}: 3355,2930,1599,1492,1466,1208,1078,1042$. Anal. Calcd for $\mathrm{C}_{13} \mathrm{H}_{17} \mathrm{ClO}_{6}$ : C, 51.24; H, 5.62. Found: C, 51.12; H, 5.68. (3,5Dimethoxyphenyl)methyl $\beta$-D-glucopyranoside (4s): ${ }^{1} \mathrm{H}-\mathrm{NMR}\left(\mathrm{D}_{2} \mathrm{O}\right)$ $\delta: 6.73(2 \mathrm{H}, \mathrm{s}), 6.58(1 \mathrm{H}, \mathrm{s}), 4.89(1 \mathrm{H}, \mathrm{d}, J=12 \mathrm{~Hz}), 4.74(1 \mathrm{H}, \mathrm{d}$ $J=12.5 \mathrm{~Hz}), 4.51(1 \mathrm{H}, \mathrm{d}, J=8 \mathrm{~Hz}), 3.94(1 \mathrm{H}, \mathrm{d}, J=12 \mathrm{~Hz}), 3.84(6 \mathrm{H}$, s), $3.70-3.75(1 \mathrm{H}, \mathrm{m}), 3.38-3.49(3 \mathrm{H}, \mathrm{m}), 3.31(1 \mathrm{H}, \mathrm{t}, J=8.5 \mathrm{~Hz})$. IR $(\mathrm{KBr}) \mathrm{cm}^{-1}: 3377,2938,2836,1596,1472,1210,1160,1038,1012$. Anal. Calcd for $\mathrm{C}_{15} \mathrm{H}_{22} \mathrm{O}_{8}$ : C, 54.54; H, 6.71. Found: C, 54.72; H, 6.80. (3,5-Dimethoxyphenyl)methyl $\beta$-D-galactopyranoside (4t): ${ }^{1} \mathrm{H}-\mathrm{NMR}$ $\left(\mathrm{D}_{2} \mathrm{O}\right) \delta: 6.74(2 \mathrm{H}, \mathrm{s}), 6.59(1 \mathrm{H}, \mathrm{s}), 4.90(1 \mathrm{H}, \mathrm{d}, J=12 \mathrm{~Hz}), 4.75(1 \mathrm{H}$, d, $J=12.5 \mathrm{~Hz}), 4.45(1 \mathrm{H}, \mathrm{d}, J=8 \mathrm{~Hz}), 3.92(1 \mathrm{H}, \mathrm{s}), 3.85(6 \mathrm{H}, \mathrm{s}), 3.77-$ $3.81(2 \mathrm{H}, \mathrm{m}), 3.61-3.69(2 \mathrm{H}, \mathrm{m}), 3.59(1 \mathrm{H}, \mathrm{t}, J=8 \mathrm{~Hz})$. IR $(\mathrm{KBr})$ $\mathrm{cm}^{-1}: 3384,2937,2878,1601,1473,1208,1157,1048,1017$. Anal. Calcd for $\mathrm{C}_{15} \mathrm{H}_{22} \mathrm{O}_{8}$ : C, 54.54; H, 6.71. Found: C, 54.43; H, 6.58 .

12) Das S. K., Reddy K. A., Mukkanti K., Carbohydr. Res., 342, 23092315 (2007).

13) Deng M., Wu Z. G., Liu X. Y., Fourth Mil. Med. Univ., 28, 15011502 (2007).

14) Yu S., Liu M., Gu X. S., Cell Mol. Neurobiol., 28, 1067-1078 (2008). 